\title{
-Originals-
}

\section{Nafamostat mesilate, a synthetic protease inhibitor, attenuated hypercoagulability in a canine model of hemorrhagic shock}

\author{
Yuichi Koido ${ }^{1)}$, Kazuyoshi Kato ${ }^{1)}$, Masumi Shimizu-Suganuma ${ }^{2)}$ \\ and Kazuhiro Shichinohe ${ }^{2)}$ \\ ${ }^{1)}$ Department of Emergency and Critical Care Medicine, Nippon Medical School \\ ${ }^{2}$ Division of Laboratory Animal Science, Nippon Medical School
}

\begin{abstract}
Hypercoagulability is known to occur in the early phase of hemorrhagic shock. The prolongation of excessive clot formation after recovery from a shock state leads to the formation of microthrombi or disseminated intravascular coagulation which disturbs microcirculation, damaging organ function. The aim of the present study is to investigate the beneficial effect of a synthetic protease inhibitor, 6-amidino-2-naphthyl p-guanidinobenzoate dimethanesulfonate (nafamostat mesilate), in the attenuation of hypercoagulability in hemorrhagic shock. A model of hemorrhagic shock that simulates the clinical course of injured patients was created in anesthetized dogs. The animals were divided into two groups: a control group (group-C, $n=9$ ) and an experimental group (group-E, $n=9$ ). Animals received saline or $0.2 \mathrm{mg} / \mathrm{kg}$ of nafamostat mesilate respectively when their mean arterial pressure declined to $50 \mathrm{mmHg}$. The serum concentration of hydroxytryptamine (5-HT), prothrombin time (PT), and activated partial thromboplastin time (APTT) were determined as indicators of platelet activity and blood coagulation.

In group-C, serum 5-HT was elevated significantly at $60 \mathrm{~min}$ after hemorrhagic shock but not so in group-E. The APTT at 30 and 60 min was shorter in group-C than in group-E. The PT at $30 \mathrm{~min}$ was also shorter in group-C. Plasma fibrin degradation products (FDP) increased at $60 \mathrm{~min}$ after the induction of shock in group-C. The results indicate that inadequate tissue perfusion in shock stimulates blood coagulation and that nafamostat mesilate might be beneficial in decreasing excessive blood coagulation. (J Nippon Med Sch 1997 ; 64: 9-15)
\end{abstract}

Key words: early phase of hemorrhagic shock, platelet aggregation, 5-Hydroxytryptamine, disseminated intravascular coagulation, nafamostat mesilate

\section{Introduction}

It is natural that blood coagulability increases in hemorrhaging patients to prevent further bleeding. However, persistence of hypercoagulability gives rise to obstruction of blood vessels by formation of thromboembolism or coagulopathy by consumption of coagulation factors ${ }^{1}$. As a consequence, defi- ciency of oxygen and nutrient supply to the tissues following a shock event may cause organ damage ${ }^{2}$. Synthetic protease inhibitors have been reported to block proteolytic enzymes in platelet aggrega. tion, coagulation and fibrinolysis ${ }^{3,4}$. Nafamostat mesilate is a synthetic antiprotease, inhibiting the

Correspondence to Yuichi Koido, Department of Emergency and Critical Care Medicine, Nippon Medical

School Hospital, 1-1-5 Sendagi, Bunkyo-ku, Tokyo 113, Japan 
activity of factors XIIa and Xa, thrombin, and plasmin as well as platelet aggregation ${ }^{5-7}$. It is expected that treatment with nafamostat mesilate in the early course of hemorrhagic shock could prevent hypercoagulability. In the present study, the authors created a model of hemorrhagic shock in dogs that simulates the pathophysiological changes that occur in the clinical shock entity and observed the effects of nafamostat mesilate on coagulation function.

\section{Materials and Methods}

Eighteen adult mongrel dogs weighing 7 to $25 \mathrm{~kg}$ were used in the present study. They received intravenous sodium pentobarbital $25 \mathrm{mg} / \mathrm{kg}$ for the induction of anesthesia. Endotracheal tubes were placed and respiration was controlled by mechanical ventilation with a tidal volume of $10 \mathrm{ml} / \mathrm{kg}$ at 20 cycles per minute. Needle electrodes were placed to monitor standard lead II electrocardiogram on a polygraph. Then, an intravenous infusion catheter was placed in the right external jugular vein to administer drugs. A catheter was inserted into the right carotid artery for continuous monitoring of arterial blood pressure. A laparotomy was performed through a midline incision to simulate an injury to the abdomen. The aorta was accessed just proximal to its bifurcation for blood sampling and for inducing hemorrhage. After all preparations for the experiment were completed, an initial blood specimen was taken via the aortic catheter, and physiological data were measured as the values for time zero.

Hemorrhagic shock was induced in each dog by withdrawing $200-550 \mathrm{~m} l$ of blood via the arterial catheter at a rate of $2 \mathrm{ml} / \mathrm{kg} / \mathrm{min}$ until the mean arterial pressure (MAP) dropped to $45-55 \mathrm{mmHg}$. In all dogs, target pressure was obtained in around fifteen minutes. The MAP was maintained at this level for 30 minutes to simulate an actual clinical hemorrhagic event.

The animals were randomly divided into two groups, control (group-C) and experimental (groupE). Each group was composed of 9 dogs. Dogs in group-C received $5 \mathrm{~m} l$ of distilled water and $2 \mathrm{~m} l$ of saline after hypotension had been maintained for 30 minutes. Animals in group-E were given $0.2 \mathrm{mg} / \mathrm{kg}$ of nafamostat mesilate dissolved in $5 \mathrm{ml}$ of distilled water and $2 \mathrm{~m} l$ of saline. The saline was used for flushing. The experimental protocol were shown in Fig. 1.

The nafamostat mesilate was kindly donated by Torii Pharmaceutical Co, Ltd, Tokyo, Japan.

Blood specimens were taken every 30 minutes after the injection of drugs or distilled water. No fluid was given throughout the experiment. Plasma 5-HT was analysed by high-performance liquid chromatography. PT was determined by the Quick

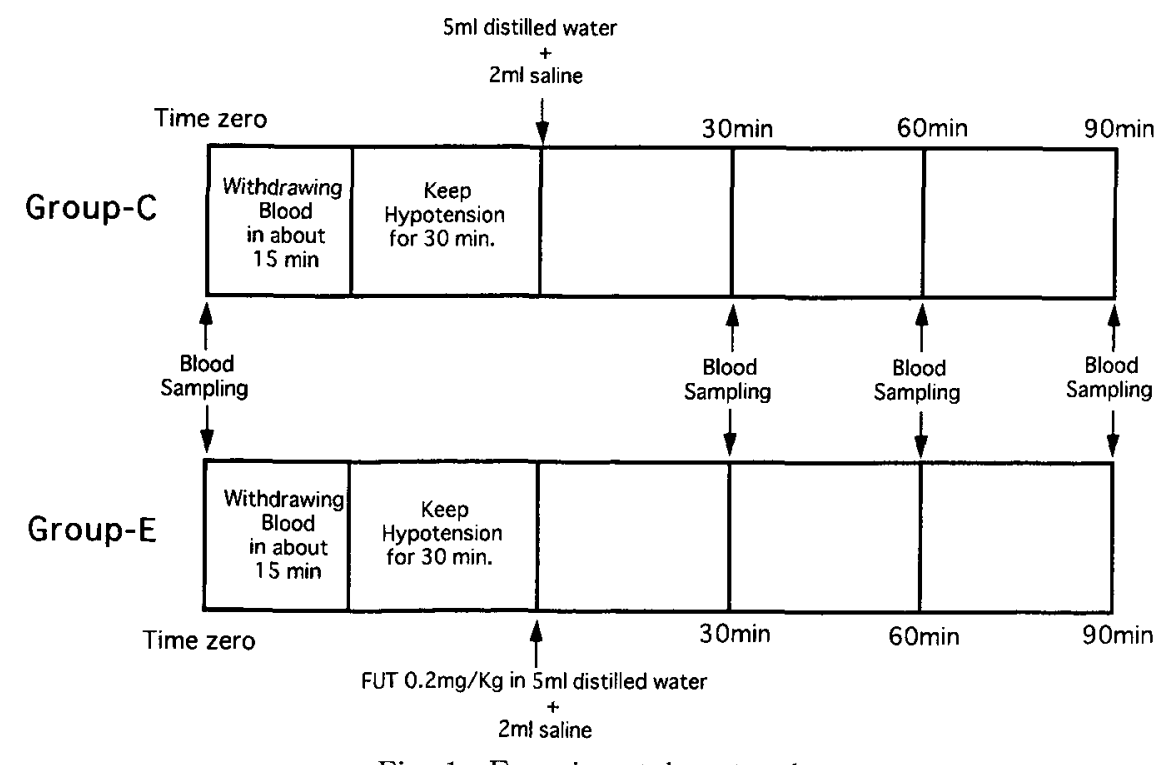

Fig. 1 Experimental protocol 
one step method and APTT by Langdell's method. Fibrin/Fibrinogen degradation products (FDP) were measured by the latex aggregation test. Arterial blood gases were analysed using a gas analyser (ABL-3, Radiometer Co, Ltd, Copenhagen).

The values obtained were expressed as mean \pm standard error of the mean (SE). The differences between the two groups were analysed with the unpaired Student's t-test. The differences between sample times were evaluated with the paired Student's t-test or Wilcoxon's signed rank test. Significance was assured if the $\mathrm{p}$ value was less than 0.05 .

\section{Results}

There were no changes in body weight, or blood pressure throughout the whole experiment between the two groups.

Hemoglobin levels dropped from $15.5 \pm 1.6 \mathrm{~g} / \mathrm{d} l$ to $11.4 \pm 2.1 \mathrm{~g} / \mathrm{d} l$ at the terminal stage of shock in group-C and from $17.8 \pm 1.3 \mathrm{~g} / \mathrm{d} l$ to $13.8 \pm 0.8 \mathrm{~g} /$ $\mathrm{d} l$ in group-E.

Arterial blood pH decreased from $7.474 \pm 0.036$ to $7.219 \pm 0.065$ in group-C and $7.569 \pm 0.046$ to $7.331 \pm 0.04$ in group-E.

There were no differences between the two groups in blood hemoglobin level and arterial blood $\mathrm{pH}$ at any observation point.

The changes in plasma 5-HT are shown in Fig. 2.

The 5-HT concentration in group-C at 60 minutes $(181 \pm 61 \mathrm{ng} / \mathrm{m} l)$ was significantly higher than that at time zero $(104 \pm 31 \mathrm{ng} / \mathrm{m} l)(\mathrm{p}<0.05)$. There were no changes in 5-HT concentration in group- $\mathrm{E}$ according to time.

The serum concentration of 5-hydroxyindoleacetic acid (5-HIAA) at 90 minutes after the induction

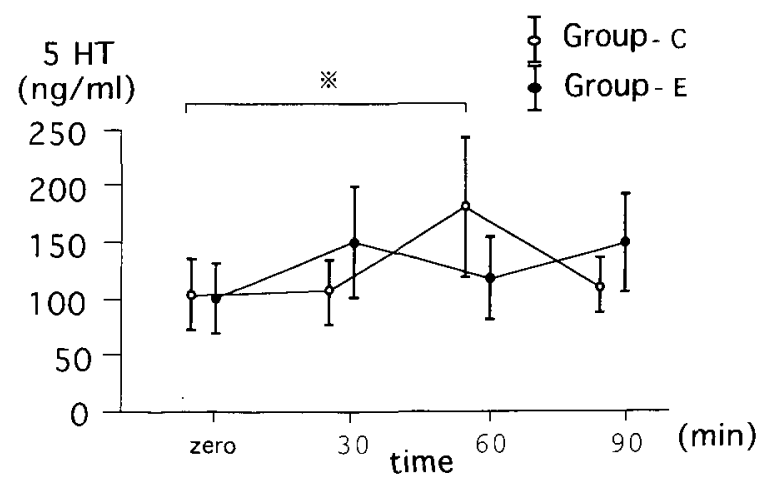

Fig. 2 Change in 5-HT concentration The concentration at 60 minutes (181 $\pm 61 \mathrm{ng} / \mathrm{ml}$ ) is significantly higher than that at time zero $(104 \pm 31$ $\mathrm{ng} / \mathrm{m} l)$ in group- $\mathrm{C}(※: \mathrm{p}<0.05)$. There were no significant changes in group-E. of hemorrhagic shock was higher than that at time zero $(\mathrm{p}<0.05)$ in group-C (time zero: $12.5 \pm 2.1 \mathrm{ng} /$ $\mathrm{m} l, 90$ minutes: $19.6 \pm 2.7 \mathrm{ng} / \mathrm{m} l)$. The concentrations of 5-HIAA were higher at 30,60 , and 90 minutes during shock than at time zero in group- $E$ $(p<0.05)$. However, no significant differences were observed between the two groups (Fig. 3).

The APTT in group-C at 30 and 60 minutes were shorter than those in group- $\mathrm{E}$ at 30 minutes (groupC: $16.6 \pm 0.5$ second; group-E: $19.8 \pm 1.0$ second; $\mathrm{p}<0.01$ ) and 60 minutes (group-C: $16.1 \pm 1.1 \mathrm{sec}$ ond; group-E: $19.7 \pm 1.1$ second; $\mathrm{p}<0.05)$ respectively (Fig. 4).

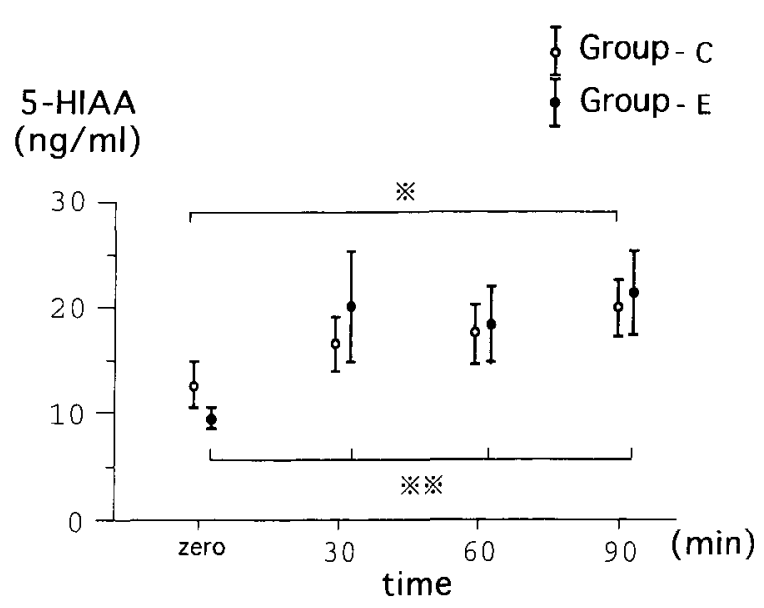

Fig. 3 Change in 5-HIAA concentration The concentration 90 minutes after the induction of hemorrhagic shock is higher than that at time zero $(\%: \mathrm{p}<$ 0.05 ) in group-C (time zero: $12.5 \pm 2.1$ $\mathrm{ng} / \mathrm{m} l, 90$ minutes: $19.6 \pm 2.7 \mathrm{ng} / \mathrm{m} l$ ). The concentration of 5 -HIAA was higher at each time than at time zero $(※ ※: \mathrm{p}<0.05)$ in group-E (time zero: $9.5 \pm 1.0 \mathrm{ng} / \mathrm{m} l, 30$ minutes: $20.1 \pm$ $5.3 \mathrm{ng} / \mathrm{ml}, 60$ minutes: $18.3 \pm 3.6 \mathrm{ng} /$ $\mathrm{m} l, 90$ minutes: $21.3 \pm 4.0 \mathrm{ng} / \mathrm{m} l$ ) 


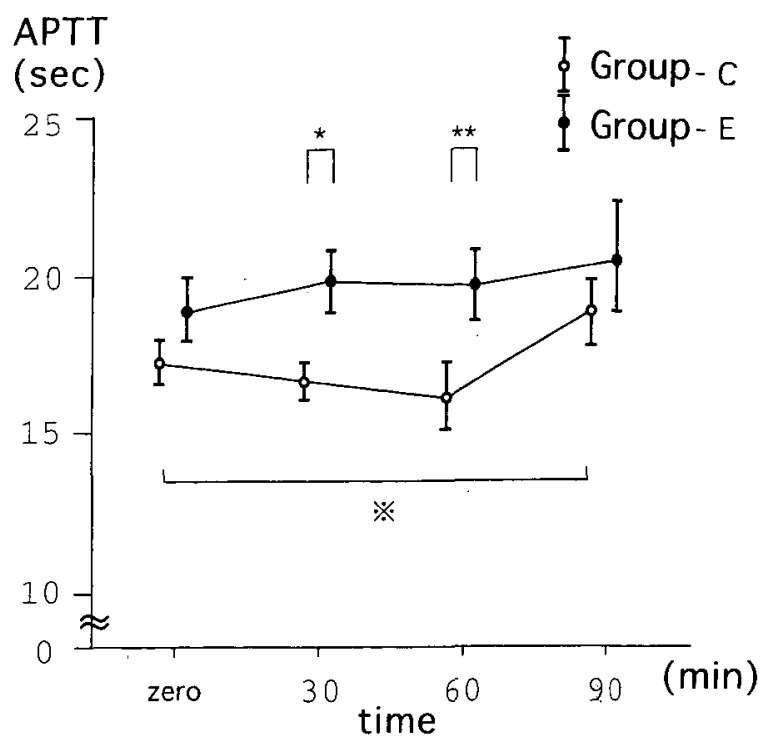

Fig. 4 Change in APTT

At 30 minutes, the APTT in group-C (16.6 \pm 0.5 second) was significantly shorter than in group-E $(19.8 \pm 1.0$ second, $\left.{ }^{*}: \mathrm{p}<0.01\right)$. There was also a significant difference between the two groups for APTT at 60 minutes (groupC: $16.1 \pm 1.1$ second, group-E: $19.7 \pm$ 1.1 second, $\left.{ }^{* *}: \mathrm{p}<0.05\right)$. In group-C, APTT at 90 minutes $(18.8 \pm 1.0 \mathrm{sec}$ ond) was significantly longer than that for time zero $(17.1 \pm 0.6$ second, $※$ : $\mathrm{p}<0.05$ )

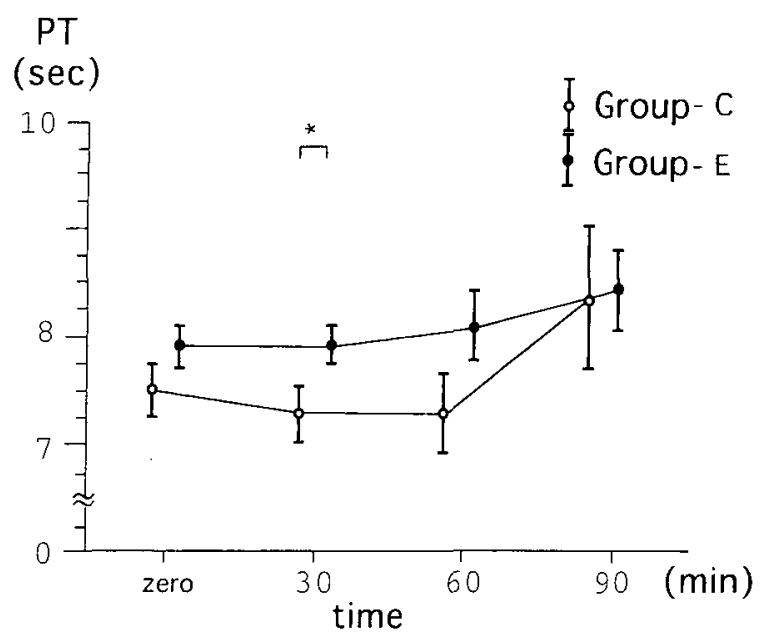

Fig. 5 Change in PT

There was a significantly difference between group-C $(7.3 \pm 0.2$ second $)$ and group-E ( $8.0 \pm 0.2$ second) at 30 minutes $(*: p<0.05)$.

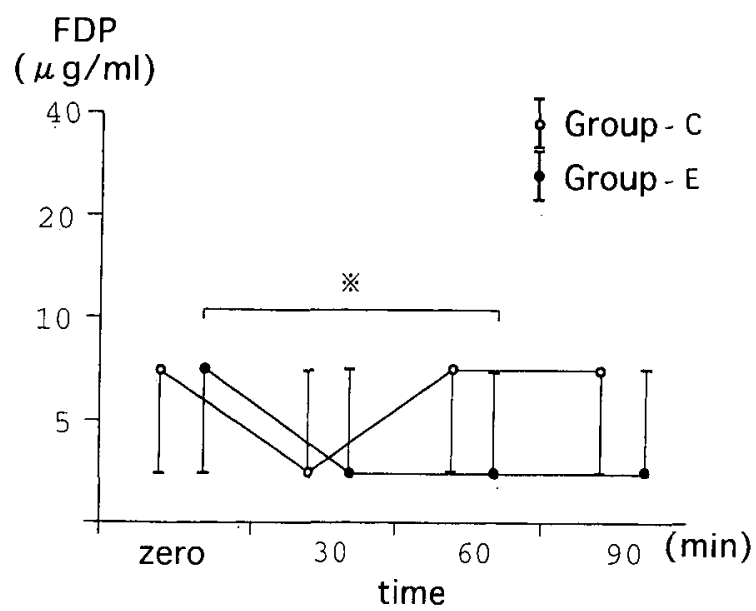

Fig. 6 Change in FDP

In group-E, FDP was decreased at 60 minutes compare with time zero $(\mathrm{p}<$ $0.05)$. No other significant differences were found.

The prothrombin time in group- $\mathrm{C}$ at 30 minutes was shorter than that in group-E (group-C: $7.3 \pm$ 0.2 second; group-E: $8.0 \pm 0.2$ second; $p<0.05$, Fig. 5).

The range of FDP at 60 minutes was significantly lower than at time zero in group-E while the range of FDP in group-C did not show any significant change (Fig. 6).

\section{Discussion}

The experimental model for hemorrhagic shock used in this study was created according to a modification of Wigger's method ${ }^{8}$ and faithfully simulates the clinical course of injured patients. In Tokyo, severely traumatized patients are rescued by emergency ambulance personnel on average 
within 15 minutes and transported to an emergency center within 30 minutes $^{9}$. The duration of bleeding in this shock model was chosen to resemble the time taken until rescue from when an emergency call to the command center of the Tokyo Fire Department is received and the 30 minute period during which hypotension was maintained was chosen to resemble the time taken for transportation to a tertiary emergency center.

In the actual clinical situation, treatment such as hemostatic intervention and fluid infusion starts when a patient arrives at an emergency center. In many other reports, experimental animals were given either crystalloid infusions or blood transfusions, which would have affected their coagulation patterns. In the present study, however, no fluids or blood products were given other than nafamostat mesilate. This gives us a rather more natural pattern of the factors involved in hemorrhagic shock which would be closer to the clinical events that occur before medical treatment is started in a traumatized patient.

There are reports that hypercoagulability occurs early after trauma ${ }^{10,11}$, but there are few reports dealing with coagulability in the early stages of hemorrhagic shock. In this experiment we look at coagulability in a model of hemorrhagic shock that was designed to simulate actual clinical circumstances.

We measured 5-HT as a marker of platelet activity. When platelets aggregate, they release 5 -HT, along with ADP and metabolites of arachidonate (e. g., thromboxane $A_{2}$ ). 5-HT, once released, further acts on 5-HT receptors in platelet membranes to enhance platelet aggregation. 5-HT also has a powerful constrictive effect on vascular smooth muscle and this, together with its effect of aggregating platelets and constricting vessels is how it aids in hemostasis ${ }^{12}$.

In this experiment, serum concentrations of 5-HT showed some increase in group- $\mathrm{C}$ while there was no change in group- $E$ after the induction of hemorrhagic shock. $90 \%$ of 5 -HT is located in the enterochromaffin cells of the gastrointestinal tract and most of the rest is found in platelets and the CNS and rarely appears in the circulation in physiologically normal situations.

The reason why 5 -HT increases in group-C is because of hemorrhagic shock, i.e., aggregated platelets are thought to release $5-\mathrm{HT}^{13,14}$. On the other hand, in group-E, nafamostat mesilate suppressed platelet aggregation and thus suppressed the release of 5 -HT.

A significant increase over time in the serum concentration of 5-HIAA, the main metabolite of 5-HT was seen, but there was no significant difference seen between the 2 groups. As the breakdown of 5-HT to 5-HIAA takes over one hour, this increase may be related to a decrease in the urinary excretion of 5-HIAA.

In the present experiment, in group-C, the APTT at 30 minutes and at 60 minutes was significantly shortened when compared with group-E. In group-C, the PT at 30 minutes was also shorter than in group-E and while not significant, there was a trend for the APTT and PT to be shorter at 30 and 60 minutes than at time 0 .

This indicates that coagulability is increased during hemorrhage and implies that nafamostat mesilate ought be able to control this as well.

Other reports also suggest that in the early phase of shock, the coagulation system becomes rather active for the first 2 hours after the induction of hemorrhagic shock, and thereafter it starts to lose its activity ${ }^{13}$.

This phenomenon of shock is a reasonable reaction to bleeding, and serves to effect hemostasis, however, it may not always be beneficial.

On one hand, excessive hypercoagulability promotes hypercoagulability. These changes in coagulability seem to occur because of the development of platelet aggregations in the microcirculation with activation of the clotting system in the early phase of hemorrhagic shock.

The prolongation of excessive clot formation after recovery from a shock state leads to the formation of microthrombi or disseminated intravascular coagulation which disturbs microcirculation, damaging organ function.

Nafamostat mesilate on its own has anti-thrombin, anti-XIIa, anti-plasmin, anti-kallikrein, antitrypsin, anti-complement and antithrombocytic properties. There have been numerous reports on its effects on coagulation ${ }^{5-7}$.

In DIC complicating sepsis, nafamostat mesilate is used commonly because it inhibits excessive 
hypercoagulability and this is thought to control the organ damage that is caused by microthrombi ${ }^{14}$. This experiment shows that nafamostat mesilate also has a corrective effect on the early hypercoagulability that occurs in hemorrhagic shock. Our results indicate that nafamostat mesilate may prove to be of potential benefit in the clinical treatment of shock.

Furthermore, changes in FDP were investigated as part of this study. In grop-C, there was a tendency for FDP to increase compaired with time zero, while the 60 minute value was significantly decreased compaired with time zero. This suggests that hypercoagulability as well as hyperfibrinolysis exist from the outset in hemorrhagic shock and that nafamostat mesilate ought to be able to suppress this hyperfibrinolysis as well.

Fibrinolysis in the early post-trauma period is under debate. A recent report involving molecular markers showed that hyperfibrinolysis can exist in the early post-trauma period ${ }^{15}$. Most reports equate the secondary fibrinolysis that occurs after coagulation with hyperfibrinolysis, but there is also a report focuses on its involvement in primary fibrinolysis.

Shimada et al. used nafamostat mesilate during and after hepatic resection and were able to decrease the incidence of transfusions and do away with FFP altogether. Because nafamostat mesilate suppresses primary fibrinolysis (the fibrinolysis that occurs as a result of extrinsic factors and stresses from hemorrhagic shock), that occurs prior to the onset of hypercoagulability ${ }^{16}$.

The results of this experiment indicate that hemorrhagic shock is implicated in fibrinolysis.

While there are many drugs that act as pure anticoagulants, in the early phase of hemorrhagic shock when hypercoagulability and primary fibrinolysis are believed to arise simultaneously, the ideal drug would be one that acts not just as an anticoagulant, but one that has anti-fibrinolytic action as well. Such a drug is nafamostat mesilate.

Hardaway reported that pure hemorrhagic shock did not cause disseminated intravascular coagulation (DIC) in experimental situations using plastic bag reservoirs ${ }^{17}$. When complicated by tissue injury however, shock is followed by DIC ${ }^{18-19}$. The initiating factor of the hypercoagulability that is caused by trauma is the release of tissue thromboplastin from damaged tissues. In our experiment, tissue damage was limited to that which is caused by a laparotomy, which is not all that severe. Nevertheless, coagulation was still activated. We believe that a major stress such as hemorrhagic shock is implicated in the development of these conditions. Clinically, in cases of severe trauma associated with hemorrhagic shock, a diagnosis of DIC is made after the resolution of shock and there are numerous cases where antiproteolytic enzymes are prescribed.

Summation: In cases of severe trauma associated with hemorrhagic shock, often there is organ damage following the resolution of shock. Organ hypoxia due to poor perfusion is thought to be the main cause of organ damage, but, our results would appear to indicate that in the early phase of hemorrhagic shock, platelet aggregation and coagulability are increased, and that microthrombi forming in organs as a result of increased coagulability may be one of the causes of organ damage. In addition, platelet aggregation and hypercoagulability could be suppressed to a certain extent by the use of nafamostat mesilate.

Acknowledgements: The authors are grateful to Prof. Yasuhiro Yamamoto for his helpful discussion.

We would also like to express our appreciation to Prof. Toshifumi Otsuka for his kind consideration.

\section{References}

1. Cate H, Brandjes DPM, Woiters HJ, Deventer SJH: Disseminated intravascular coagulation: Pathophysiology, diagnosis, and treatment. New Horizons 1993; 2: 312-323.

2. Shoemaker WC: Relation of oxygen transport patterns to the pathophysiology and therapy of shock states. Intensive Care Med 1987; 13: 230-243.

3. Ohno H, Kambayashi J, Chang SW, Kōsaki G: FOY:
[Ethyl p-(6-guanidinohexanoyloxy) benzoate] methanesulfonate as a serine proteinase inhibitor. II. In vivo effect on coagulofibrinolytic system in comparison with heparin or aprotinin. Thromb Res 1981; 24: 445-452.

4. Taenaka N, Shimada Y, Hirata T, Nishijma MK, Takezawa J, Yoshiya I, Kambayashi J: Gabexate mesilate (FOY) therapy of disseminated intravasu- 
cular coagulation due to sepsis. Critical Care Med 1983; 11: 735-738.

5. Fuji S, Hitomi Y: New synthetic inhibitors of $\mathrm{Clr}, \mathrm{Cl}$ esterase, thrombin, plasmin. kallikrein and trypsin. Biochim Biophys Acta 1981; 661: 342-345.

6. Aoyama T, Ino Y, Ozeki M, Oda M, Sato T, Koshiyama Y, Suzuki S, Fujita M: Phamacological studies of FUT-175, nafamostat mesilate. I. Inhibition of protease activity in in vitro and in vivo experiments. Japan J Pharmacol 1984; 35: 203-227.

7. Hitomi Y, Ikari N, Fujii S: Inhibitory effect of a new synthetic protease inhibitor (FUT-175) on the coagulation system. Hemostasis 1985; 15: 164-168.

8. Werle JM, Cosby RS, Wiggers CJ: Observation on hemorrhagic hypotention and hemorrhagic shock. Am J Physiol 1942; 136: 401-420.

9. Rescue Activities 1994. Annual report of the Tokyo Fire Department. Tokyo Fire Department 1995.

10. Kapsch DN, Metzler M, Harrington M, Mitchell FL, Silver D: Fibrinolytic response to trauma. Surgery 1984; 95: 473-478.

11. Risberg B, Medegard A, Heideman M, Gyzander E, Bundsen $P$, Oden M, Nilsson A: Early activation of humoral proteolytic systems in patients with multiple trauma. Crit Care Med 1986; 14: 917-925.

12. Garrison JC: Histamine, bradykinin, 5-hydroxy- triptamine and antagonists. In the pharmacological basis of therapeutics. 8th ed. Gilman AG, Rall TW, Nies AS, 1990; pp 592-599. Taylor Peds, Pergamon Press inc, New York.

13. Hollenberg NK: Serotonin and vascular responses. Annu Rev Pharmacol Toxicol 1988; 28: 41-49.

14. Houston DS, Vanhoutte PM: Serotonin and the vascular system: Role in health and disease, and implications for therapy. Drugs 1986; 31: 149-163.

15. Gando S, Tedo I. Kubota M: Posttrauma coagulation and fibrinolysis. Crit Care Med 1992; 20: $595-600$.

16. Shimada M, Matsumata T, Shirabe K, Kamakara $\mathrm{T}$, Taketomi A, Sugimachi K: Effect of nafamostat mesilate on coagulation and fibrinolysis in hepatic resection. J Am Coll Surg 1994; 178: 498-502.

17. Hardaway RM, Johnson DG, Elovits MJ: Influence of trauma and hemolysis on hemorrhagic shock in dogs. J Trauma 1964; 4: 673-676.

18. Hardaway RM, Mechanism of traumatic shock. Surg Gynecol Obstet 1980; 151: 65-69.

19. Hardaway RM, Williams $\mathrm{CH}$ : Influence of steroids on hemorrhagic and traumatic shock. J Trauma 1987; 27: $667-670$.

(Received, April 30, 1996)

(Accepted for publication, September 4, 1996) 